\title{
Commentary on William Thomson's "Pitch Frames as Melodic Archetypes"
}

\author{
DAVID TEMPERLEY \\ Eastman School of Music, University of Rochester
}

\begin{abstract}
While the concept of the perceptual "pitch frame" resembles leading theories of pitch structure in music in some respects, it contains some innovative elements that are discussed in this commentary. Additionally, the commentary focuses on the question of whether the "pitch frame" is a temporal or atemporal construct.
\end{abstract}

Submitted 2006 April 12; accepted 2006 April 19.

KEYWORDS: perception, tonality, modality, melody, harmony, pitch space, temporal

WILLIAM Thomson's "Pitch Frames as Melodic Archetypes" offers a thoughtful and suggestive new perspective on tonal organization. While there is certainly some merit to Thomson's "pitch frame" idea, I wish to raise two concerns about it. One is that the theory seems ambivalent with regard to the issue of temporality: is the pitch frame essentially a temporal or atemporal construct? The second, related, issue is that it is unclear to what extent the pitch frame idea is something really new, as opposed to simply a repackaging of ideas already familiar from other theories. I believe there are original elements in Thomson's idea, but these have not yet been articulated as clearly as they could be. I now elaborate these points.

Thomson's pitch frame consists of a series of pitches represented in music notation, with different rhythmic symbols representing different degrees of structural importance. There are two ways that such a representation might be construed. One is as an atemporal representation, indicating pitch levels that maintain a certain status of stability or importance throughout a melody. The other construal is as a representation of actual events in time; construed as such, the pitch frame clearly does not represent all the events of a melody, but only the most important ones, and uses notation to make further distinctions of structural importance among these. There are precedents for both the temporal and atemporal conceptions of the pitch frame. As an atemporal conception, it brings to mind constructs such as Krumhansl's "keyprofile" (1990) and Lerdahl's "basic pitch space" (2001), shown in Figure 1:

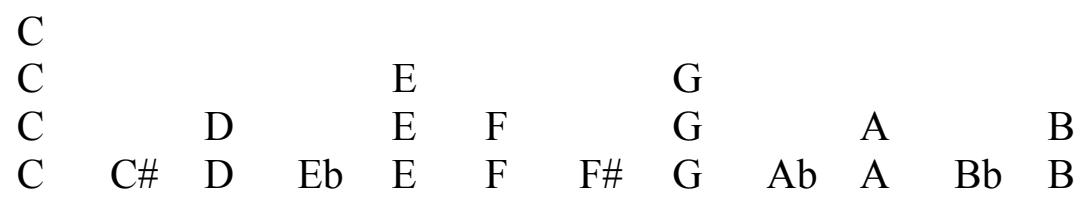

Fig. 1. Lerdahl's basic pitch space (2001), oriented around I of C major.

(There are also differences, however, as I will discuss below.) Each of these is an atemporal tonal hierarchy which governs the pitch organization of an entire melody. As a temporal conception, the pitch frame seems similar to a Schenkerian analysis (or Lerdahl's "prolongational analysis"). (This distinction between temporal and atemporal construals brings to mind Bharucha's (1984) distinction between a "tonal hierarchy" and an "event hierarchy".)

So which is the right construal of the pitch frame - temporal or atemporal? One's first impression is that it is a temporal construct. If it were atemporal, the ordering of pitches in Thomson's pitch frame representations would seem to be arbitrary: one would expect some standard convention to be used-for 
example, arranging the pitches in descending registral order. And indeed, many of Thomson's tonality frames reflect this. But then, what about his Figure 4? In that case, the pitches are arranged bottom to top. In figures 6 and 10, neither a top-to-bottom or bottom-to-top ordering is consistently followed. Thus the ordering of pitches in the tonality does represent something - and it seems reasonable to assume that it reflects temporal ordering (as the ordering of notes in music notation usually does!). But this is a very different way of interpreting the pitch frame. Now, something like the frame in Thomson's Figure 5 is not simply an atemporal framework, but rather an analysis of the structurally important events in the melody - an assertion of an underlying structural pattern "Eb5-C5-Bb4-G4-Eb4-C4." However, the temporal construal of the pitch frame also encounters problems. Consider Thomson's Figure 2: In this case, the tonality frame ends with G4. Yet it would seem quite implausible to posit an underlying structural melody ending with G4 this case; the final resting point of the melody is surely A4. Similar points could be made about Thomson's Figures 6, 7, and 10, where the sequence of pitches in the tonality frame hardly constitutes a convincing "reduction" of the melody.

It seems to me, then, the tonality frame does not quite know what it wants to be. My advice to Thomson would be to focus on the atemporal construal. We already have a fairly well-established theory of event hierarchies: Schenkerian analysis. Construed temporally, Thomson's pitch frames are a lot like Schenkerian analyses, and it is not clear that the tonality frame idea adds much beyond this. As for atemporal "tonal hierarchies": As mentioned, Thomson's frames are rather similar to other proposals of this type, especially Lerdahl's "basic space". However, there are some interesting differences. Lerdahl's basic space represents pitch-classes; by contrast, Thomson's tonality frame represents pitches in specific registers. In this way, Thomson's theory captures the fact that-for example- "London Bridge" (Thomson's Figure 1) is focused around a particular F-C fifth, whereas Lerdahl's theory does not. Lerdahl's "basic space" is also a fairly general construct: a certain configuration of the space is claimed to characterize a particular musical idiom. By contrast, Thomson's frames appear to be more specific to individual pieces. For example, for Thomson's Figure 5, Lerdahl might posit a "pentatonic triadic space"- a kind of space, shown below in Figure 2, that I would argue characterizes blues in general (as well as a good deal of rock and other styles):

\section{$\mathrm{C}$ $\begin{array}{lllllll}\mathrm{C} & \mathrm{Eb} & & \mathrm{G} & & \\ \mathrm{C} & \mathrm{Eb} & \mathrm{F} & \mathrm{G} & & \mathrm{Bb}\end{array}$ $\begin{array}{llllllllllll}\mathrm{C} & \mathrm{C} \# & \mathrm{D} & \mathrm{Eb} & \mathrm{E} & \mathrm{F} & \mathrm{F} \# & \mathrm{G} & \mathrm{Ab} & \mathrm{A} & \mathrm{Bb} & \mathrm{B}\end{array}$}

Fig. 2. A Lerdahlian "pentatonic-triadic" space

According to this framework, F and B (the 4 and flat-7 scale degrees) are both scalar, non-triadic pitches. But Thomson's pitch frame captures that, in this particular song, Bb has a stability and importance above that of F.

Because it is registrally specific, and because it is brings out distinctions of stability or importance between pitches that are specific to pieces (going beyond the general features of the style), Thomson's theory differs from Lerdahl's. The two theories are complementary: Lerdahl's captures a hierarchy of pitchclasses, and applies to a musical style as a whole; Thomson's captures a hierarchy of specific pitches that pertains to specific pieces. It seems to me that this is where the original insight, and the potential contribution, of Thomson's theory resides.

In closing, I must comment on a few remarks in the introductory section of Thomson's paper. Thomson pulls no punches in expressing his opinions about what he perceives as unexamined assumptions of music theory. For example, he criticizes the division of Western music into "modal" and "tonal" eras, finding it a simplistic and unhelpful distinction. This, I feel, is a bit of a straw man. I think everyone, at least today, acknowledges that the distinction between modal and tonal music is highly problematic: the shift from one system to the other was gradual, much music shares elements of both, and in any case the two systems have important elements in common, such as organization around a tonal center or focal pitch. But the distinction is a useful one nonetheless. (Does Miles Davis's "So What" really sound "suspiciously 
like simple old C major"?!) I am more sympathetic with Thomson's attack on what he calls the "noconcept/no-percept" assumption: The idea that it is invalid to analyze music using theoretical concepts that were not part of the composer's explicit vocabulary. (For example, some criticize the use of Roman numeral symbols in analyzing Bach for this reason.) This is, indeed, a pernicious notion in music theory and Thomson is quite right to challenge it. The entire premise of cognitive science is that there is much going on in our minds at an unconscious level that cannot readily be articulated; this applies to musicians and composers of all eras. Indeed, to accept the "no-concept/no-percept" idea would seem to rule out the possibility of any original theory about music of the past!

Thomson also takes issue with models of music perception that "count notes." Here, he presumably has in mind theories of key identification — advanced most famously by Krumhansl (1990) but also by a number of other authors - which involve tallying the frequency of occurrence of different pitchclasses. As one practitioner of this approach, I take issue with Thomson here. The fact is that models of key perception based on this principle have performed extremely well in tests of key identification (Krumhansl 1990; Temperley 2001, 2004). This does not of course prove that human perception works in this way, but it is certainly shows the computational viability of such an approach. While Thomson may be right that human key perception gives more attention to the ordering and arrangement of pitches (what he calls "parametric kinetics"), there has been a notable lack of concrete proposals in this direction. If human tonality perception relies on "parametric kinetics," how exactly does this work? It seems to me to be incumbent on critics of the "note-counting" approach to put forth a credible, testable alternative.

\section{REFERENCES}

Bharucha, J. J. (1984). Anchoring effects in music: The resolution of dissonance. Cognitive Psychology, 16, 485-518.

Krumhansl, C. (1990). Cognitive Foundations of Musical Pitch. Oxford: Oxford University Press.

Lerdahl, F. (2001). Tonal Pitch Space. Oxford: Oxford University Press.

Temperley, D. (2001). The Cognition of Basic Musical Structures. Cambridge, MA: MIT Press.

Temperley, D. (2004). Bayesian models of musical structure and cognition. Musicae Scientiae, 8, $175-205$.

Thomson, W. (2006). Pitch frames as melodic archetypes. Empirical Musicology Review, 1, 85-102. 